\title{
Comparison of clinical effect of CT diagnosis and X-ray Plain film diagnosis in children with Acute Osteomyelitis during emergency treatment
}

\author{
Jing $\mathrm{FAN}^{1}$, Yanyan $\mathrm{GUO}^{2}$, Jin $\mathrm{LI}^{3}$, $\mathrm{Li} \mathrm{HE}^{2}$, Junfen $\mathrm{ZHOU}^{4 *}$ []
}

\begin{abstract}
Background: Acute osteomyelitis has great harmfulness. To conduct comparative analysis on the difference of clinical effect of CT diagnosis and X-ray plain film diagnosis in children with acute osteomyelitis during emergency treatment, to provide guidance for the diagnosis and treatment of this disease. Methods: The study subjects were 32 children with acute osteomyelitis. All the children were examined by X-ray and CT before surgery. Image characteristics and detection rates of bone destruction, changes in periosteum and cortex were compared. Funding: X-ray scanning image: for the children with acute osteomyelitis in the early stage, the symptoms were demonstrated as that the submuscular space disappeared, and the boundary between subcutaneous and muscle tissues was blurred. CT scanning image: the images in early stage showed hyperemia and edema of soft tissue, with a slightly lower density of lesion, disappearance of interfascicular space, and a low-density abscess cavity in the center. The detection rate of bone destruction in CT scan was obviously higher than that in plain X-ray, in terms of bone destruction in special morphology, bone shell destruction in residual expansion, circular bone destruction, and peripheral bone defect, and the detection rate of periosteum and cortex changes was higher than that in plain X-ray, The diagnostic sensitivity and specificity of CT in children with acute osteomyelitis during emergency treatment were better than that of X-ray plain film. Interpretation: $\mathrm{CT}$ scan has good diagnostic accuracy, sensitivity and specificity during emergency treatment of this disease.
\end{abstract}

Keyword: children with acute osteomyelitis; emergency treatment; CT examination; X-ray plain film; diagnosis; clinical effect.

Practical Application: CT scan has good diagnostic accuracy, sensitivity and specificity for children with acute osteomyelitis during emergency treatment.

\section{Introduction}

Acute hematopoietic osteomyelitis is also known as acute osteomyelitis, which is not common in pediatric clinic but has great harmfulness. Most children with acute osteomyelitis are infected with pyogenic bacteria through hematogenous invasion of the connective tissue of the bone marrow to cause inflammatory response, while a few were spread from adjacent soft tissue infection or secondary to open fractures (Funk \& Copley, 2017). In recent years, it's shown in the investigations that the incidence of acute osteomyelitis in children has an obvious rising trend, which may be related to the increase of trauma cases, abuse of antibiotics and drug resistance of pathogenic bacteria (McBride et al., 2018). Failing to be treated timely and effectively, the bone structure of children may be damaged and lead to disability, and the infection may spread to the whole body, seriously endangering the safety of life; Some patients may turn into chronic osteomyelitis with prolonged disease course, which may affect the nutrition and growth of children (Waddell et al., 2017). This disease has always been one of the difficult pediatric clinical problems, especially non-traumatic acute osteomyelitis whose symptoms are similar with myeloma, which is more prone to misdiagnosis or missed diagnosis, and delay the treatment.

The early diagnosis during emergency treatment of acute osteomyelitis in children is of great significance for the selection of treatment plan and prognosis effect. It has been reported that delayed diagnosis and treatment may lead to the disability rate and mortality rate of $20 \%$ and $1 \%$ in children with acute osteomyelitis (de Graaf et al., 2017). Imaging examination is a main means to diagnose various orthopedic diseases and also an important method to diagnose acute osteomyelitis in children. X-ray plain film scanning is a traditional imaging diagnostic methods, which mainly makes use of a certain wavelength and frequency of X-ray and interaction between the energy conversion occurs, thereby to obtain images from part of human body, the fractures and degenerative bone disease, arthritis, widely used in diagnosing vertebral body shape change, but the image data obtained is relatively limited, so is the accuracy of identification of some minor structural change (Mandell et al., 2017). CT scanning is a computerized tomography technology developed on the basis

${ }^{1}$ Medical Administration Department, Wuhan Children's Hospital (Wuhan Maternal and Child Healthcare Hospital), Tongji Medical College, Huazhong University of Science \& Technology, Wuhan, China

${ }^{2}$ Department of Radiology, Wuhan Children's Hospital (Wuhan Maternal and Child Healthcare Hospital), Tongji Medical College, Huazhong University of Science \& Technology, Wuhan, China

${ }^{3}$ Department of Ultrasonography, Wuhan Fourth Hospital; Puai Hospital, Tongji Medical College, Huazhong University of Science and Technology, China

${ }^{4}$ Department of Nuclear Medicine, The Central Hospital of Wuhan, Tongji Medical College, Huazhong University of Science and Technology, Wuhan, China

*Corresponding author: zhoujunfen26@aliyun.com 
of plain X-ray scanning, which uses accurate collimating X-ray bundles, $\gamma$-ray, ultrasonic waves and etc. to obtain images, which shortens the time spent on scanning and produces clear images and etc (Yu et al., 2019). This study will conduct comparative analysis on the difference of clinical effect of CT diagnosis and $\mathrm{X}$-ray diagnosis in children with acute osteomyelitis during emergency treatment, to provide guidance for the diagnosis and treatment of this disease.

\section{Methods}

\subsection{General Data}

The study subjects were 32 children with acute osteomyelitis who were admitted to our hospital from June 2017 to December 2019 and confirmed through pathology of puncture operation. The retrospective analysis was launched after being reviewed and approved by the Ethics Committee of the hospital. The clinical data of sick children were collected, including male: 24 patients (75.00\%), female: 8 patients (25.00\%), the age ranged from 3 months to 12 years old, average age: $(10.02 \pm 1.85)$ years, duration of course of disease: $0-28 \mathrm{~d}$, mean course of disease: $(5.63 \pm 2.24) \mathrm{d}$; Clinical symptom manifestations of the included children: fever in 32 patients, local pain in 29 patients, local swelling in 23 patients, skin flushing in 15 patients; Pathologically, the lesions were all located in long bones, with 17 patients of femur (53.13\%) and 15 patients of tibia (46.87\%). The self-control study was conducted.

Inclusion criteria: Determined and diagnosed (Yeddes et al., 2017) according to relevant criteria for acute osteomyelitis specified in Surgery $\left(4^{\text {th }}\right.$ version$)$, confirmed through pathology of puncture operation; examined with X-ray plain film and CT scan before operation, complete image data; the age of sick children ranged from 3 months to 12 years old; The parents of sick children know and agree to the content and purpose of this study.

Exclusion criteria: Children with osteoporosis and other severe bone diseases; Not treated with antibiotics; The pathology of puncture operation confirmed that the lesion site was not long bone; Lack of clinical data and unsigned study consent.

\subsection{Research Methods}

\subsubsection{X-ray plain film scanning}

Take proper positions from the children, use digital X-ray plain film machine (Philips Digital Diagnost TH+VT500mA) to perform normal and lateral scanning of the diseased bone and adjacent joint bone, and the tissue image was processed with DR system to obtain the image scanning results.

\subsubsection{CT scanning examination}

Take proper positions from the children, Use 64-raw spiral CT (Siemens) for conventional CT scanning and enhanced CT scanning on the bone at the lesion positions and adjacent joint bones, with a thickness of $4.0 \mathrm{~mm}$ and a spacing of $4.0 \mathrm{~mm}$. The scanning range included the full length of the lesion and $10.0 \mathrm{~mm}$ above and below the lesion, and axial images of bone window and soft tissue window were obtained for analysis and diagnosis.

\subsection{Evaluation Index}

(1) Image data were collected, and the image characteristics of plain X-ray and CT scanning in children with acute osteomyelitis at different stages were summarized through observation and analysis; (2) The pathological diagnosis results were taken as the gold standard, and the two diagnostic methods were recorded by the imaging department of our hospital and a senior physician to compare the detection rates of bone destruction, periosteum and cortical changes of each type. (3) Compare and analyze the detection rate, sensitivity and specificity of the two diagnostic methods in emergency treatment of children with acute osteomyelitis.

\subsection{Statistical Analysis}

The statistical software SPSS 21.0 was used to analyze and process all the study data. The measurement data were expressed as mean \pm standard deviation (' $x \pm s$ ), the counting data as percentage (\%) and inter-group was inspected with " $\mathrm{t}$ " and Chi-square. $\mathrm{P}<0.05$ indicates the difference to be statistically significant.

\section{Results}

\subsection{Characteristic Analysis of X-ray Plain Scanning Image}

$X$-ray scanning image: for the children with acute osteomyelitis in the early stage, the symptoms were demonstrated as that the submuscular space disappeared, and the boundary between subcutaneous and muscle tissues was blurred, the bone trabeculae were loose and fuzzy upon the course of disease more than one week. Some children showed bone destruction, blurred edges, or low density or even transparent areas, the bone cortex gradually absorbed diffusion. The X-ray plain film scanning image of acute suppurative osteomyelitis of tibia is referred in Figure 1: the superior tibia is damaged.

\subsection{CT Characteristic Analysis of CT Scanning Image}

The CT images of acute osteomyelitis in children were characterized by: soft tissue hyperemia and edema in early stage of disease, the lesion showed slightly low density, the intermuscular tract space disappeared, low-density abscess cavity could be seen in the center, and abscess wall was found in the surrounding ring of soft tissue, and the enhanced CT scan showed ring enhancement. Bone destruction areas showed irregular marginal limited low-density shadow, or there were fluid areas, accompanied by cortical disruption, and the density of bone marrow cavity increased slightly. The CT signs of axial bone mineral density did not change significantly, the periosteum density increased, and the thickness of soft tissue between the skin showed an uneven low-density shadow. The CT scanning image of acute suppurative osteomyelitis of tibia is referred in Figure 2: the superior tibia is damaged.

\subsection{Comparison of Detection Rate of All Types of Bone Destruction in the Two Diagnostic Methods}

The data details are shown in Table 1. The detection rate of bone destruction in CT scan was obviously higher than that in 


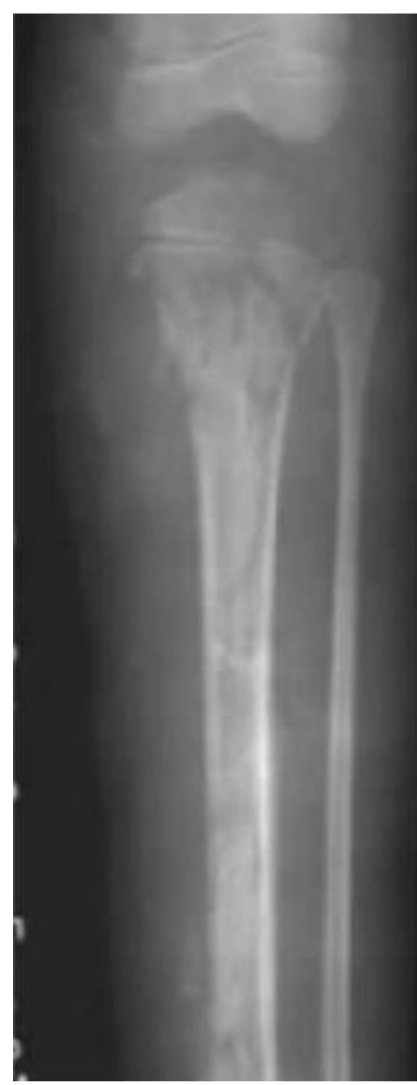

Figure 1. X-ray plain film scanning image of acute suppurative osteomyelitis of tibia: the superior tibia is damaged.

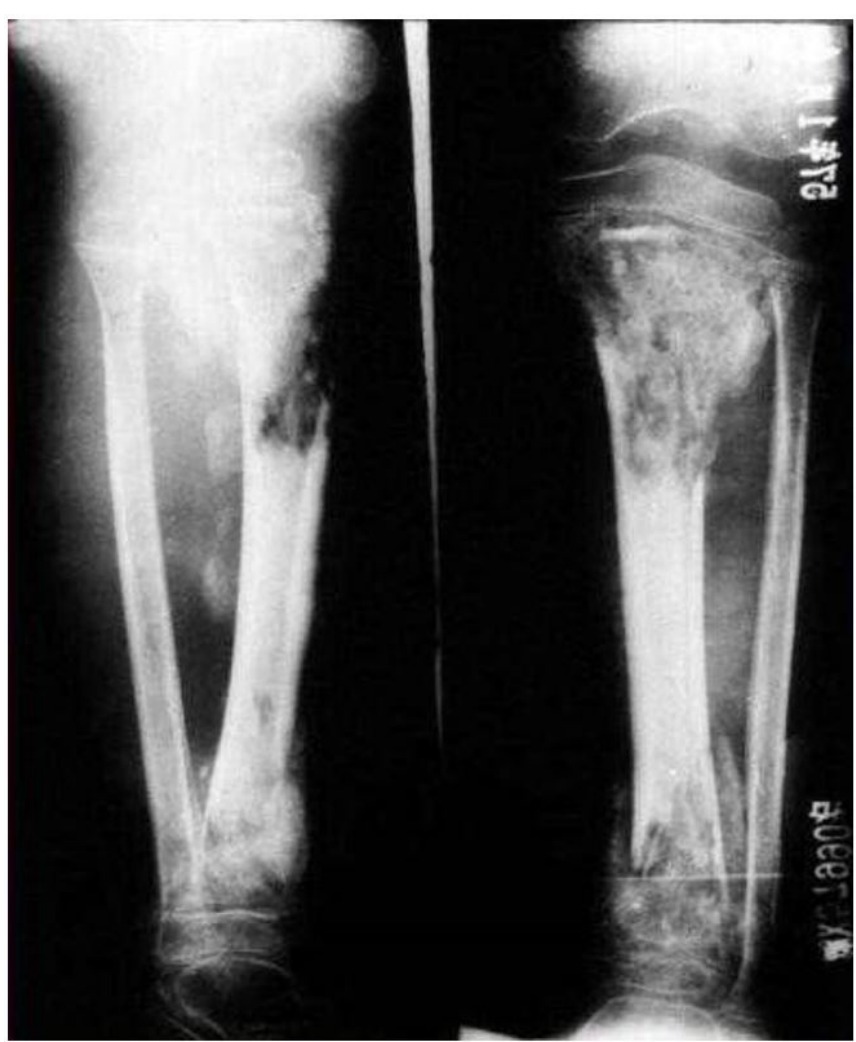

Figure 2. Scanning image of acute suppurative osteomyelitis of tibia: the superior tibia is damaged. plain X-ray, in terms of bone destruction in special morphology, bone shell destruction in residual expansion, circular bone destruction, and peripheral bone defect was higher than that in plain $\mathrm{X}$-ray, with statistically significant difference $(\mathrm{P}<0.05)$.

\subsection{Comparison of Detection Rate of Periosteum and Cortex in the Two Diagnostic Methods}

The data details are shown in Table 2. The detection rate of changes in periosteum and cortex by CT scanning was higher than that by plain $\mathrm{X}$-ray plain scanning, and the difference was statistically significant $(\mathrm{P}<0.05)$.

\subsection{Comparison of Sensitivity and Specificity in the Two Diagnostic Methods}

The data details are shown in Table 3. The diagnostic sensitivity and specificity of CT in children with acute osteomyelitis during emergency treatment were better than that of X-ray plain film, and the difference was statistically significant $(\mathrm{P}<0.05)$.

\section{Discussion}

Acute osteomyelitis is a bone-infected disease with low clinical incidence. However, the younger children are in the stage of rapid growth and development, and the vascular anatomy of bones has certain specificity, so the incidence of acute osteomyelitis is significantly higher than that of adults (Willegger et al., 2017). Blood-borne infection is the major cause of infantile acute osteomyelitis induced, with men more than women, about $40 \%$ of the patients in sepsis or soft tissue purulent infection, and pathogenic bacteria usually give priority to staphylococcus aureus. In recent years, there is also a strong white staphylococcus hemolytic toxicity, occasionally visible salmonella species, pneumococcal or other fester bacterium, and most of the pathogenic bacteria are resistant to penicillin and streptomycin (Khurana et al., 2017). Pustules, gingival abscesses and upper respiratory tract infections are common root causes of acute osteomyelitis in children (Turnbull et al., 2017). With the increase of trauma, the abuse of antibiotics and pathogenic bacteria produces drug resistance, and the clinical cases of acute osteomyelitis in children also increase (Marzec et al., 2017). If the treatment is delayed, the mortality and disability rate of this disease will increase significantly, which may cause serious complications and even turn into chronic osteomyelitis, hindering the normal growth and development of children. The accurate diagnosis in early stage is the key to reduce the mortality and disability rate of acute osteomyelitis in children and improve the prognosis.

Osteomyelitis occurs primarily at the lower end of femur and the upper end of tibia, followed by the upper end of femur, humerus and the distal radius, and may also occur in other bones (Zhou et al., 2018). The symptoms and signs of children vary with the severity of infection, the location of disease, the scope of inflammation, the course of disease, age, the resistance of body and other clinical manifestations, which can be divided into sepsis type, complicated arthritis type, localized destruction or bone abscess type (Jung et al., 2019). Sepsis-type acute osteomyelitis in children accounts for $80 \%$, the children show high fever, coma, 
Fan et al.

Table 1. Comparison of Detection Rate of All Types of Bone Destruction in the Two Diagnostic Methods.

\begin{tabular}{|c|c|c|c|c|c|}
\hline Type & Pathology & CT scanning [n (\%) ] & X-ray Plain Scanning [n (\%) ] & $\mathrm{x}^{2}$ Value & P Value \\
\hline Bone destruction in special morphology & 12 & $11(91.67)$ & $3(25.00)$ & 91.439 & 0.001 \\
\hline Bone shell destruction in residual expansion & 9 & $7(77.78)$ & $0(0.00)$ & 127.279 & 0.001 \\
\hline Circular bone destruction & 8 & $6(75.00)$ & $3(37.50)$ & 28.571 & 0.001 \\
\hline Peripheral bone defect & 8 & $6(75.00)$ & $2(25.0)$ & 50.000 & 0.001 \\
\hline Sinus changes & 5 & $5(100.00)$ & $4(80.00)$ & 22.222 & 0.001 \\
\hline Sclerotic edge bone destruction & 2 & $2(100.00)$ & $2(100.00)$ & - & - \\
\hline Total & 44 & $37(84.09)$ & $14(31.82)$ & 56.062 & 0.001 \\
\hline
\end{tabular}

Table 2. Comparison of detection rate of Periosteum and Cortex in the Two Diagnostic Methods.

\begin{tabular}{|c|c|c|c|c|c|}
\hline Type & Pathology & CT scanning [n (\%) ] & X-ray Plain Scanning [n (\%) ] & $\mathrm{x}^{2}$ Value & P Value \\
\hline Periosteum hyperplasia & 9 & $7(77.78)$ & $4(44.44)$ & 23.386 & 0.001 \\
\hline Periosteum triangle & 3 & $2(66.67)$ & $2(66.67)$ & 0.000 & 1.000 \\
\hline $\begin{array}{l}\text { Cortical thickening/focal } \\
\text { sclerosis }\end{array}$ & 2 & $2(100.00)$ & $0(0.00)$ & 200.000 & 0.001 \\
\hline Dead bone & 1 & $0(0.00)$ & $0(0.00)$ & - & - \\
\hline Total & 15 & $11(73.33)$ & $6(40.00)$ & 22.620 & 0.001 \\
\hline
\end{tabular}

Table 3. Comparison of Sensitivity and Specificity in the Two Diagnostic Methods.

\begin{tabular}{lccc}
\hline Diagnostic Method & Detection Rate & Sensitivity & Specificity \\
\hline CT scanning & $81.36 \%$ & $74.85 \%$ & $83.29 \%$ \\
X-ray plain scanning & $33.90 \%$ & $40.32 \%$ & $45.06 \%$ \\
$\mathrm{X}^{2}$ value & 46.123 & 24.408 & 31.785 \\
P value & 0.001 & 0.001 & 0.001 \\
\hline
\end{tabular}

delirium and other symptoms, toxic shock may occur under severe conditions, local symptoms of the injured limb persistent severe pain or tenderness, accompanied by peripheral swelling, invaded bone disease can be single bone or multiple bones; There are also a few children with systemic symptoms as the main manifestation, and local signs of diseased bone are late, so early detection of bone lesions is needed clinically (Aydin et al., 2018). Imaging technology plays an important role in diagnosis of various orthopedic diseases. Traditional X-ray and CT scans are especially valuable in diagnosis of fractures, osteoarthritis, rheumatism, inflammatory and degenerative bone diseases, bone and joint tumors, and spinal morphological changes, besides, a large number of studies have shown that compared with plain $\mathrm{X}$-ray, CT scan can obtain more skeletal and musculoskeletal system data and clearer image data (Ma et al., 2018).

The 32 children with pediatric acute osteomyelitis diagnosed by pathology were retrospectively analyzed in this study. The image characteristics of lesion and surrounding bones through X-ray scanning: no change of bone substance in early stage, the submuscular space disappeared, and the boundary between subcutaneous and muscle tissues was blurred, the bone trabeculae were loose and fuzzy upon the course of disease more than one week. Some children showed bone destruction, blurred edges, or low density or even transparent areas, the bone cortex gradually absorbed diffusion. The image characteristics of lesion and surrounding bones through CT scanning: early indications were hyperemia and edema of soft tissue, disappearance of intermuscular space, and low density abscess cavity in the center; around $6 \mathrm{~d}$ after onset, the lesion showed a slightly lower density, with abscess walls in the surrounding ring soft tissue, and enhanced CT scan showed ring enhancement; about $10 \mathrm{~d}$ after onset, periosteal reaction was observed, and bone destruction was seen within 2 weeks, showing an irregular marginal localized low-density shadow, or a fluid zone, accompanied by cortical disruption and a slight increase in the density of bone marrow cavity. The $\mathrm{CT}$ signs of axial bone mineral density did not change significantly, the periosteum density increased, and the thickness of soft tissue between the skin showed an uneven low-density shadow. The detection rate of CT scan on various types of bone destruction and changes in periosteum and cortex was significantly higher than that of X-ray film, indicating that CT scan would obtain clearer image data of lesions during emergency treatment of acute osteomyelitis in children, which is conducive to assisting doctors to make accurate differential diagnosis. In addition, CT scan has good sensitivity and specificity in early-stage diagnosis of this disease, indicating that this imaging method can accurately reflect the pathological process and characteristics of acute osteomyelitis in children. Although puncture operation pathological biopsy is the gold standard for diagnosis of acute osteomyelitis in children, this method is traumatic, complicated and time-consuming. Therefore, it is not suitable for emergency treatment and may delay the best time for treatment; X-ray plain film and CT scan are relatively easy to operate, which can directly show the characteristics of skeletal muscle tissue at the diseased positions of children, which is more suitable for emergency treatment. Thanks to the higher image definition and more comprehensive data acquisition, the application value of CT scanning is better than conventional plain X-ray scanning.

In conclusion, CT scanning and X-ray plain film scanning scan have certain differences in image characteristics at different 
periods during the progression of acute osteomyelitis in children; CT scanning is of favorable diagnostic accuracy, sensitivity and specificity during the emergency treatment of acute osteomyelitis in children, maintaining higher application value.

\section{References}

Aydın, N., Kocaoğlu, B., Sarığlu, E., Tok, O., \& Güven, O. (2018). The comparison of arthroscopic acromioplasty with and without acromioclavicular coplaning. Ulusal Travma ve Acil Cerrahi Dergisi, 24(3), 274-277. PMid:29786825.

de Graaf, H., Sukhtankar, P., Arch, B., Ahmad, N., Lees, A., Bennett, A., Spowart, C., Hickey, H., Jeanes, A., Armon, K., Riordan, A., Herberg, J., Hackett, S., Gamble, C., Shingadia, D., Pallett, A., Clarke, S. C., Henman, P., Emonts, M., Sharland, M., Finn, A., Pollard, A. J., Powell, C., Marsh, P., Ballinger, C., Williamson, P. R., Clarke, N. M., \& Faust, S. N. (2017). Duration of intravenous antibiotic therapy for children with acute osteomyelitis or septic arthritis: A feasibility study. Health Technology Assessment, 21(48), 1-164. http://dx.doi. org/10.3310/hta21480. PMid:28862129.

Funk, S. S. \& Copley, L. A. B. (2017). Acute Hematogenous Osteomyelitis in children: Pathogenesis, diagnosis, and treatment. The Orthopedic Clinics of North America, 48(2), 199-208. http://dx.doi.org/10.1016/j. ocl.2016.12.007. PMid:28336042.

Jung, S. W., Oh, S. H., Lee, I. S., Byun, J. H., \& Lee, J. H. (2019). In situ gelling hydrogel with anti-bacterial activity and bone healing property for treatment of Osteomyelitis. Tissue Engineering and Regenerative Medicine, 16(5), 1-12. http://dx.doi.org/10.1007/ s13770-019-00206-x. PMid:31624703.

Khurana, A., Chhawra, S., Gupta, R., \& Kumar, S. (2017). Osteomyelitis of Fibula rare case with various differential diagnosis. Journal of Orthopaedic Case Reports, 7(3), 59-62. PMid:29051882.

Ma, L., Hu, P., Wu, Z. Q., \& Song, X. Z. (2018). Clinical application of vacuum sealing drainage combined with chymotrypsin in the treatment of post-traumatic mandibular osteomyelitis. Zhonghua Kou Qiang Yi Xue Za Zhi, 53(2), 123-124. PMid:29429233.

Mandell, J. C., Khurana, B., Smith, J. T., Czuczman, G. J., Ghazikhanian, V., \& Smith, S. E. (2017). Osteomyelitis of the lower extremity: pathophysiology, imaging, and classification, with an emphasis on diabetic foot infection. Emergency Radiology, 25(2, Suppl. 1), 175188. http://dx.doi.org/10.1007/s10140-017-1564-9. PMid:29058098.

Marzec, N. S., Nelson, C., Waldron, P. R., Blackburn, B. G., Hosain, S., Greenhow, T., Green, G. M., Lomen-Hoerth, C., Golden, M., \& Mead, P. S. (2017). Serious bacterial infections acquired during treatment of patients given a diagnosis of Chronic Lyme Disease - United States. MMWR. Morbidity and Mortality Weekly Report, 66(23), 607-609. http://dx.doi.org/10.15585/mmwr.mm6623a3. PMid:28617768.

McBride, S., Thurm, C., Gouripeddi, R., Stone, B., Jaggard, P., Shah, S. S., Tieder, J. S., Butcher, R., Weiser, J., Hall, M., Keren, R., \& Landrigan, C. P. (2018). Comparison of empiric Antibiotics for Acute Osteomyelitis in children. Hospital Pediatrics, 8(5), 280-287. http:// dx.doi.org/10.1542/hpeds.2017-0079. PMid:29626010.

Turnbull, C., Young, L., \& Lowery, S. (2017). Oral vs. Intravenous antibiotics for the treatment of acute bacterial Osteomyelitis in the veteran population. Open Forum Infectious Diseases, 4(Suppl. 1), S96-S96.

Waddell, J., Mcculloh, R., Goldman, J., Lee, B., \& Teachout, W. (2017). Comparative analysis of initial antibiotic dosing among healthy weight, overweight, and obese children with Osteomyelitis. Open Forum Infectious Diseases, 4(Suppl. 1), S91-S92.

Willegger, M., Kolb, A., Windhager, R., \& Chiari, C. (2017). Acute haematogenous osteomyelitis in children: Diagnostic algorithm and treatment strategies. Der Orthopade, 46(6), 1-15. PMid:28534215.

Yeddes, I., Meddeb, I., Bouchoucha, S., Slim, I., Zgolli, H., Mhiri, A., \& Ben Slimene, M. F. (2017). The bone scintigraphy in the diagnosis and management in children with febrile osteoarticular pain. $L a$ Tunisie Medicale, 95(2), 109-114. PMid:29424869.

Yu, L., Yu, G., Deng, K., \& Wang, G. (2019). Asymmetric limb lengthening in the treatment of tibial hemimelia caused by osteomyelitis. Case Reports in Medicine, 98(3), e14031. PMid:30653110.

Zhou, J., Zhou, X. G., Wang, J. W., Zhou, H., \& Dong, J. (2018). Treatment of osteomyelitis defects by a vancomycin-loaded gelatin $/ \beta$-tricalcium phosphate composite scaffold. Bone \& Joint Research, 7(1), 4657. http://dx.doi.org/10.1302/2046-3758.71.BJR-2017-0129.R2. PMid:29330343. 